\title{
A NOTE ON THE CONDITIONAL CHROMATIC POLYNOMIAL
}

\author{
by V. VOLOSHIN
}

(Received 20 April, 1992)

In this note we consider a finite graph without loops and multiple edges. The colouring of a graph $G$ in $\lambda$ colours is the colouring of its vertices in such a way that no two of adjacent vertices have the same colours and the number of used colours does not exceed $\lambda[1,4]$. Two colourings of graph $G$ are called different if there exists at least one vertex which changes colour when passing from one colouring to another.

Definition. A function $P\left(G_{0} \subset G, \mu, \lambda\right)$ is called a conditional chromatic polynomial of a graph $G$ if it expresses the number of colourings of $G$ in $\lambda$ colours provided that the given subgraph $G_{0} \subset G$ is coloured in $\mu \leq \lambda$ colours.

We suppose that $P\left(G_{0} \subset G, \mu, \lambda\right)=0$ if $\mu>\lambda$ or $G_{0} \not G$. Note that $P\left(G_{0} \subset G, \lambda, \lambda\right)$ coincides with usual chromatic polynomial [1, p. 352]. If $G_{0}$ and $G_{1}$ are two subgraphs of $G$ with the same vertex set, then $P\left(G_{0} \subset G, \mu, \lambda\right)=P\left(G_{1} \subset G, \mu, \lambda\right)$.

If $F_{n}$ is a full (also known as 'complete') graph on $n$ vertices, $k \leq n$, then $P\left(F_{k} \subset F_{n}, \mu, \lambda\right)=\mu^{(k)}(\lambda-k)^{(n-k)}$, where $\mu^{(k)}=\mu(\mu-1)(\mu-2) \ldots(\mu-k+1)$. In particular $P\left(F_{k} \subset F_{n}, \lambda, \lambda\right)=\lambda^{(k)}(\lambda-k)^{(n-k)}=\lambda^{(n)}$.

If $E_{n}$ is empty (also known as 'null' or 'totally disconnected' graph on $n$ vertices) then $P\left(E_{k} \subset E_{n}, \mu, \lambda\right)=\mu^{k} \lambda^{n-k}$, in particular $P\left(E_{k} \subset E_{n}, \lambda, \lambda\right)=\lambda^{n}$.

Let $G=(X, V),|X|=n$ be a graph and $G_{0}=\left(X_{0}, V_{0}\right), X_{0} \subset X_{0}\left|X_{0}\right|=m$ be an induced subgraph of $G$. Let also $x, y \in X$ be two non-adjacent vertices in $G$. We construct the graph $G_{1}$ from $G$ by joining $x$ and $y$ by an edge and the graph $G_{2}$, obtained from $G$ by contracting $x$ and $y$ into single vertex. Then we can observe that the following equality is true:

$$
P\left(G_{0} \subset G, \mu, \lambda\right)=P\left(G_{0}^{1} \subset G_{1}, \mu, \lambda\right)+P\left(G_{0}^{2} \subset G_{2}, \mu, \lambda\right),
$$

where $G_{0}^{1}=G_{0}^{2}=G_{0}$ if $x, y \notin X_{0}$, and $G_{0}^{1}, G_{0}^{2}$ are the subgraphs induced respectively by $X_{0}, X_{0} \cup\{x, y\}$ otherwise. It is so because $P\left(G_{0} \subset G_{2}, \mu, \lambda\right)$ equals the number of colourings for which $x$ and $y$ have the same colour.

If we perform this operation further as much as possible we obtain

$$
P\left(G_{0} \subset G, \mu, \lambda\right)=\sum_{i=1}^{t} P\left(F_{k_{i}} \subset F_{n_{i}}, \mu, \lambda\right) .
$$

Since $P\left(F_{k} \subset F_{n}, \mu, \lambda\right)=P\left(F_{k}^{\prime} \subset F_{n}, \mu, \lambda\right)$ for any two $k$-vertex complete subgraphs $F_{k}, F_{k}^{\prime}$ of $F_{n}$, we can write

$$
P\left(G_{0} \subset G, \mu, \lambda\right)=\sum_{i=1}^{m} \sum_{j=i}^{n} \alpha_{i j} P\left(F_{i} \subset F_{j}, \mu, \lambda\right) .
$$

In order to clear up the meaning of coefficients $\alpha_{i j}$ we consider one more class of colourings. A colouring of a graph is called an $i$-colouring, if exactly $i$ colours are used, but

Glasgow Math. J. 36 (1994) 265-267. 
two $i$-colourings are assumed to be distinct if there is a pair of vertices $x$ and $y$ in $G$ which have the same colour for one of these colourings and different colours for the other [4, p. 18]. Let $g_{i}(G)$ be the number of $i$-colourings of $G$. By analogy introduce the number $g_{i j}\left(G_{0} \subset G\right)$ which equals the number of $j$-colourings of $G$ provided that subgraph $G_{0} \subset G$ is $i$-coloured $(i \leq j)$.

It is evident that

$$
g_{i j}\left(F_{k} \subset F_{n}\right)= \begin{cases}1, & \text { if } i=k, j=n \\ 0 & \text { otherwise. }\end{cases}
$$

Since $g_{k l}\left(G_{0} \subset G\right)=g_{k l}\left(G_{0}^{1} \subset G_{1}\right)+g_{k l}\left(G_{0}^{2} \subset G_{2}\right)$ as in (1), after repeating this procedure as often as possible we have

$$
g_{k l}\left(G_{0} \subset G\right)=\sum_{i=1}^{m} \sum_{j=i}^{n} \alpha_{i j} g_{k l}\left(F_{i} \subset F_{j}\right) .
$$

Consequently, $\alpha_{i j}=g_{i j}\left(G_{0} \subset G\right), i=1, \ldots, m ; j=i, \ldots, n$.

Now we can state the following

Theorem. For any graph $G=(X, Y),|X|=n$ and its subgraph $G_{0}=\left(X_{0}, V_{0}\right), X_{0} \subset X$, $\left|X_{0}\right|=m$, the conditional chromatic polynomial has the following form:

$$
P\left(G_{0} \subset G, \mu, \lambda\right)=\sum_{i=1}^{m} \sum_{j=i}^{n} g_{i j}\left(G_{0} \subset G\right) \mu^{(i)}(\lambda-i)^{(j-i)} .
$$

One can derive many properties of $P\left(G_{0} \subset G, \mu, \lambda\right)$ which are the generalization of usual properties of $P(G, \lambda)[2,3]$. For example when considering the case $E_{k} \subset E_{n}$ we obtain an "algebraic" result:

COROLLARY [3]. The coefficients in the expansion of $\mu^{m} \lambda^{n-m}(1 \leq m \leq n)$ into the powers of form $\mu^{(i)}(\lambda-i)^{(j-i)}$ are the generalizations of Stirling's numbers and are calculated by the formula:

$$
V_{i j}^{n m}= \begin{cases}S(i, n), & \text { if } i=j, j=1,2, \ldots, n \\ 0 & \text { otherwise } \\ j V_{i j}^{m, n-1}+V_{i, j-1}^{m, n-1}, i=1, \ldots, m ; j=1, \ldots, n, & \text { if } m<n, \\ 0 & \text { if } i>m \text { or } j>n \text { or } m>n \text { or } i>j,\end{cases}
$$

where $S(i, n)$ are the Stirling numbers of the second kind.

Proof. $V_{i j}^{m n}=g_{i j}\left(E_{m} \subset E_{n}\right)$. If $m<n$ consider any $x \in E_{n} \backslash E_{m}$. If there exists $y \in E_{n}$, $y \neq x$ which is coloured in the same colour, for example $j$, then the number of such colourings is $j g_{i j}\left(E_{m} \subset E_{n-1}\right)$, otherwise the number of colourings will be $g_{i j-1}\left(E_{m} \subset E_{n-1}\right)$.

The initial fragment of $V_{i j}^{m n}$-numbers for $m=1,2,3,4, n=1,2,3,4$ is shown in the 
following table:

\begin{tabular}{|c|c|c|c|c|}
\hline$n$ & 1 & 2 & 3 & 4 \\
\hline 1 & 1000 & - & - & - \\
\hline 2 & 1100 & 1000 & - & - \\
& 0000 & 0100 & & \\
\hline \multirow{2}{*}{3} & 1310 & 1100 & 1000 & \\
& 0000 & 0210 & 0300 & - \\
& 0000 & 0000 & 0010 & \\
\hline \multirow{3}{*}{4} & 1761 & 1310 & 1100 & 1000 \\
& 0000 & 0451 & 0630 & 0700 \\
& 0000 & 0000 & 0031 & 0060 \\
& 0000 & 0000 & 0000 & 0001 \\
\hline
\end{tabular}

The conditional chromatic polynomial $\lambda$ greatly extends the class of polynomials which may be considered as chromatic. For example polynomial $3 \lambda^{4}$ may be interpreted as $P\left(E_{1} \subset E_{5}, 3, \lambda\right)$ and so on. Finally, if $\gamma\left(G_{0}\right)$ is chromatic number of $G_{0}$, then the conditional chromatic number $\gamma_{G_{0}}(G)$ is the smallest integer $\lambda$ such that $P\left(G_{0} \subset\right.$ $\left.G, \gamma\left(G_{0}\right), \lambda\right) \neq 0$ (cf. [4, p. 261]).

\section{OPEN PROBLEMS}

1. Find a necessary and sufficient condition for polynomial of two variables to be the conditional chromatic polynomial of some graph.

2. Find the meaning of coefficients and roots of the conditional chromatic polynomial.

3. Find, for a graph $G$, all subgraphs $G_{i}$ and $G_{j}$ such that

$$
P\left(G_{i} \subset G, \mu, \lambda\right)=P\left(G_{j} \subset G, \mu, \lambda\right) .
$$

4. When does the equality $P\left(G_{1} \subset G_{2}, \mu, \lambda\right)=P\left(G_{3} \subset G_{4}, \mu, \lambda\right)$ imply isomorphism of graphs $G_{1}$ and $G_{3}$ or $G_{2}$ and $G_{4}$ respectively?

\section{REFERENCES}

1. C. Berge. Graphs and Hypergraphs (North-Holland, Amsterdam, 1973).

2. V. Voloshin. Conditional chromatic polynomial. In Research on applied mathematics and informatics, Kishinev, Science, 1990, 16-19 (in Russian).

3. V. Voloshin. Conditional chromatic polynomial. In Methods and programs of resolving of optimization problems on graphs and networks. Part 1. Theses of reports (Novosibirsk, 1989 (in Russian)).

4. A. Zykov. Fundamentals of graph theory (BCS Associates, Moscow, and Idaho USA, 1990).

Department of Mathematical Cybernetics

Moldova State University,

Kishinev 277009, Moldova 